\title{
Development of E-Book for the Basics of Observing the Sky Object
}

\author{
Wiraporn Maithon* \\ Department of Physics and General Science, Faculty of \\ Science and Technology \\ Chiang Mai Rajabhat University \\ Chiang Mai, Thailand \\ *wiraporn@g.cmru.ac.th
}

\author{
Sommkorn Chaiwarakorn \\ Faculty of Science Technology and Agriculture Yala \\ Rajabhat University \\ Yala, Thailand \\ sommkorn.c@yru.ac.th
}

\begin{abstract}
This electronic book (e-book) involves the horizontal coordinate system explanation and the basis of the sky object observation. We analyzed the astronomy cause description of the undergraduate level in many Universities and developed this e-book for the undergraduate students. The performance content accuracy of the book and the appropriateness of the question were checked by the experts for investigating the ItemObjective Congruence Index (IOC). The e-book was tried out with the undergraduate students. The samples users in this work are the undergraduate students who must learn astronomy in Chiang Mai Rajabhat University and Yala Rajabhat University. The result shows that the average satisfaction of students who performed this e-book is a very satisfactory level $(4.80 \pm 0.40$, overall). Therefore, the e-book for the basics of observing sky objects can be used as a teaching and learning media to develop effective teaching and learning which encourages learners to participate in teaching and learning via social media.
\end{abstract}

Keywords-E-Book, horizontal system, sky object observation

\section{INTRODUCTION}

Educational technology is applying new techniques, methods, concepts, devices, and tools to solve the learning problems of education. Including the expansion and improving the quality of teaching too. Therefore, it means that educational technology covers three things: the introduction of new tools for teaching and learning, the production of new teaching materials, and the use of new techniques or applications. The materials equipment and techniques of anyone or a combination of systems are created to produce and develop education in mass-communication such as printing media, broadcasting radio, and television. Furthermore, information technology educational development, computer, internet, multimedia-telecommunications network, telephone, and other communication are achieving the learning process according to the daily life-learners at all times. Moreover, educational technology improved the student's achievement learning and better than the previous [1-3].

In this research, we are interested to study and develop an e-book for the undergraduate learner. We choose the
Astronomy for the first case study and design an e-book about the basics of observing sky object. The samples are the undergraduate students in general Science and Physics Education major in Chiang Mai Rajabhat University and Yala Rajabhat University to find satisfaction.

\section{Methodology}

The conceptual design in this study as shown in Fig.1.

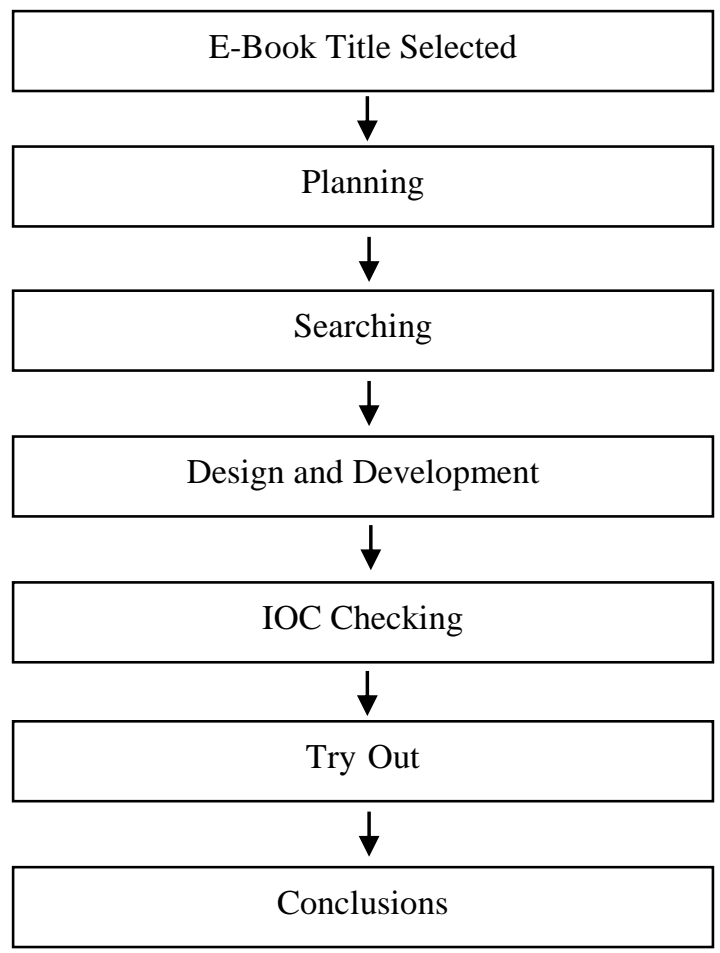

Fig. 1. Conceptual diagram of the e-book development

In the conceptual diagram from Fig.1, firstly, we surveyed about the Astronomy description in many Rajabhat Universities and set the topic for e-book development. Secondly, we planned the scope in this work and lastly, we 
searched and collected for all of the data. The e-book for the basics of observing sky objects was designed, developed, and was Index of Consistency (IOC) checked by the 3 experts. We adjusted this e-book from the expert's information and try out with the undergraduate students.

The case study in this work was astronomy about the basics of observing sky objects in undergraduate students. They must review the basic of the celestial sphere; the astronomical coordinates system involves the horizontal coordinate [4]. The position in this coordinate is explaining by azimuth and altitude that as shown in Fig.2.

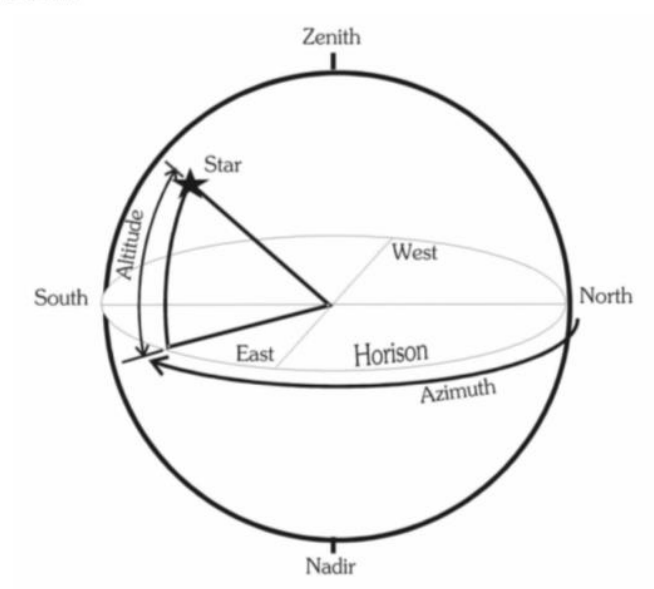

Fig. 2. Horizontal coordinate.

The azimuth was computed to look like the circle from the north to the east, the south, the west. The altitude was calculated from the horizontal to the zenith. This coordinate is easiest to measure the position of the sky object such as the Orion nebular that as shown in Fig.3.

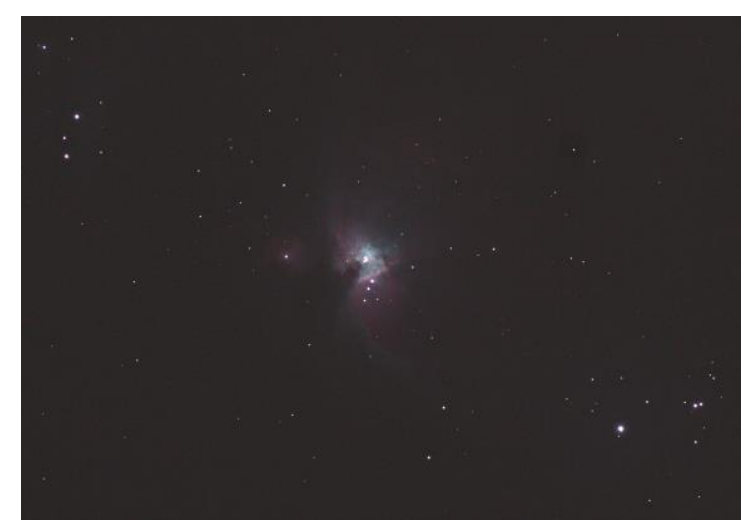

Fig. 3. Example of an Orion nebula.

The Orion nebular is a nebula in the Orion constellation, so this e-book has to explain the constellation too. Everyone who learns from this e-book should understand the basics of the sky object observation; the horizontal coordinate and the constellation include the star map that as shown in Fig.4.

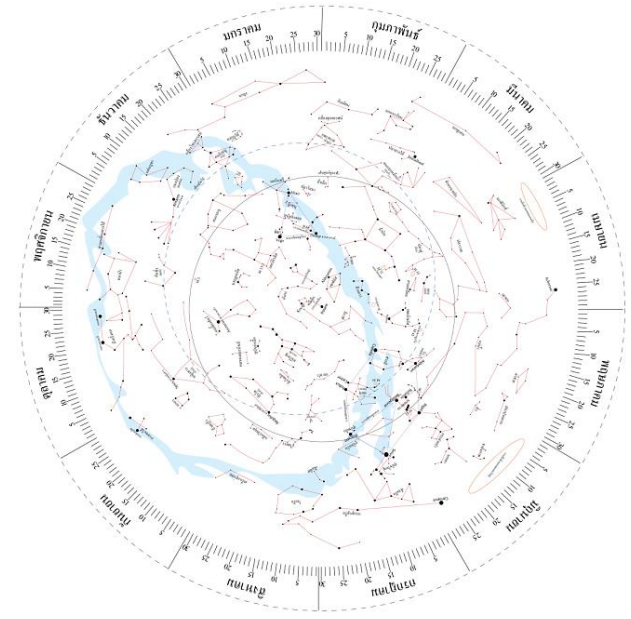

Fig. 4. Star map.

After the development of the e-book for the basics of observing the sky object was completed. The IOC was checked by the experts in 4 main points,

- Contents.

- Image and language.

- Alphabets, background, and color.

- Electronics media.

The e-book for the basics of observing sky objects in this work was used to try out by the third-year students in general Science and Physics Education major at Chiang Mai Rajabhat University and Yala Rajabhat University. In this session, the information about the satisfaction of the users in 5 categories was gathered.

- The e-book for the basics of observing sky objects is interesting.

- This e-book is not complex, easy to use, and not confused.

- The user can understand and apply to observation.

- This e-book makes them want to learn and want to research in the future.

- Overall

- The satisfaction was founded from the mean value of the samples that

Level 5 is very good,

Level 4 is good,

Level 3 is medium,

Level 2 is low, 
Level 1 is the adjustment

Finally, the satisfaction criterion in this study as shown in Table I.

\section{TABLE I. THE CRITERION OF THE SATISFACTION}

\begin{tabular}{|l|l|l|}
\hline Level & \multicolumn{1}{|c|}{ the point between } & \multicolumn{1}{|c|}{ satisfaction } \\
\hline 5 & $4.50-5.00$ & very much \\
\hline 4 & $3.50-4.49$ & Much \\
\hline 3 & $2.50-3.49$ & Medium \\
\hline 2 & $1.50-2.49$ & Low \\
\hline 1 & $1.00-1.49$ & very low \\
\hline
\end{tabular}

The statistical analysis in this research is the IOC, mean and Standard Deviation (S.D.),

- the e-book was considered about the content validity, objectives, clarity, accuracy, and appropriateness by the IOC with 3 experts. The points must more than 0.5 each [5]. The IOC equation is, [6]

$$
\mathrm{IOC}=\frac{\sum \mathrm{R}}{\mathrm{N}}
$$

When IOC is the index of consistency between the objectives and content,

$\mathrm{R}$ is the expert's point, and

$\mathrm{N}$ is the number of experts

The specifying criteria as follows,

$\begin{array}{ll}\text { Appropriate } & \text { The point is }+1, \\ \text { Uncertain } & \text { The point is } 0 \text {, and } \\ \text { Inconsistent } & \text { The point is }-1 \text {. }\end{array}$

- Mean that is to find the middle of the score. It will give you an overview of the students, so

$$
\overline{\mathrm{x}}=\frac{\sum \mathrm{x}}{\mathrm{n}}
$$

n

When $\overline{\mathrm{X}}$ is the mean

$\mathrm{X}$ is the satisfaction points of students each, and

$n$ is the total of students.

- S.D. that shows the distribution of points. If it is much, it means the points are very different, so,

$$
\text { S.D. }=\sqrt{\frac{\sum(\mathrm{x}-\overline{\mathrm{x}})^{2}}{\mathrm{n}-1}}
$$

When S.D. is the standard deviation,

$\mathrm{x}$ is the satisfaction points of students each, and $\mathrm{n}$ is the total of students.

\section{RESULTS}

The Example of the basics of observing sky objects as shown in Fig.5.
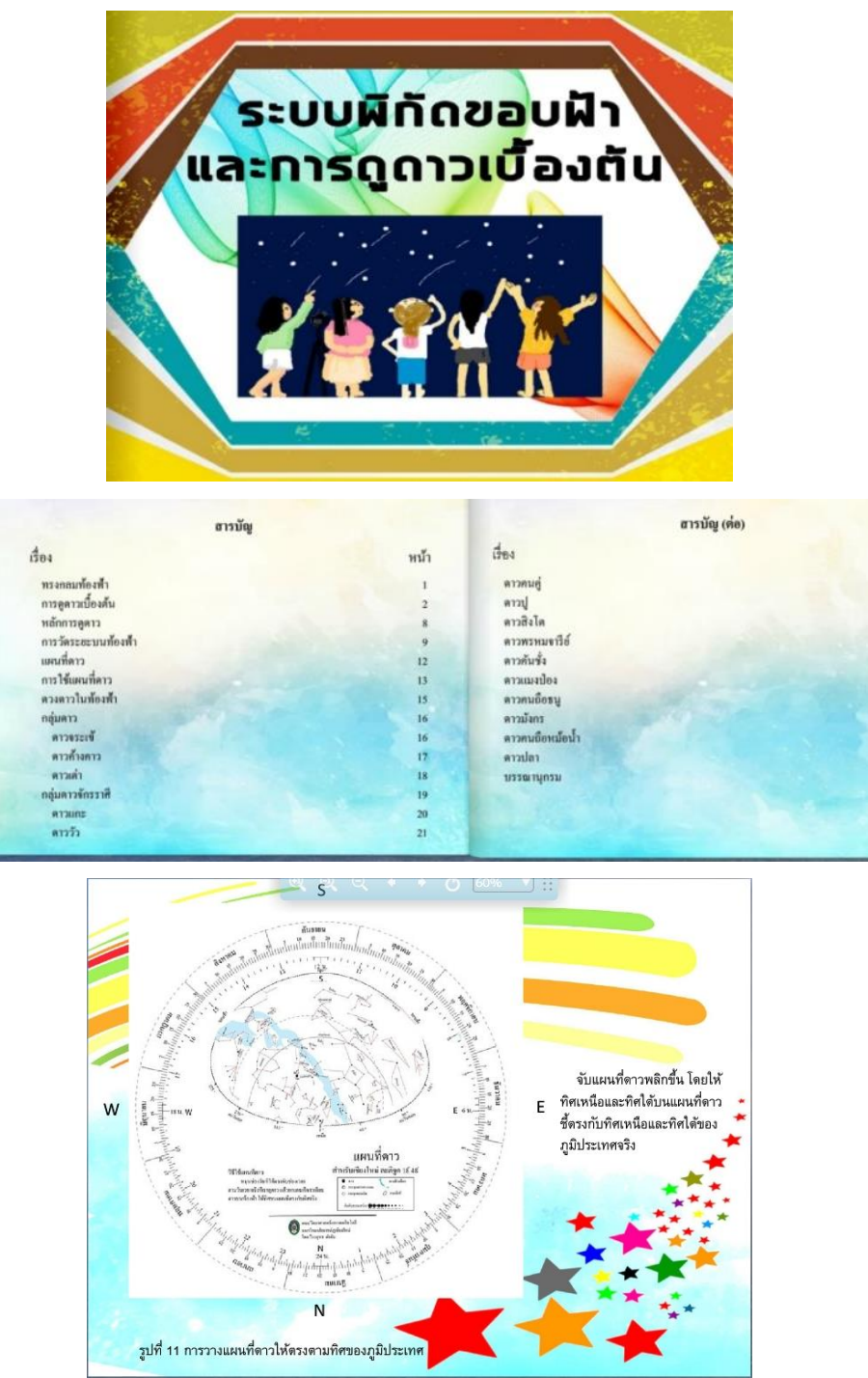

Fig. 5. Example of an e-book for the basics of observing sky objects.

This e-book was examined by 3 experts in 4 main points that as shown in Table 2 
TABLE II. THE IOC FROM EXPERT

\begin{tabular}{|l|l|}
\hline \multicolumn{1}{|c|}{ Item } & \multicolumn{1}{|l|}{ IOC } \\
\hline Contents & 1 \\
\hline 1. The correctness of the content. & 0.67 \\
\hline 2. The description of the content is clear. & 1 \\
\hline 3. The sequence of the presentation is appropriate. & 1 \\
\hline 4. The presentation is interesting. & 1 \\
\hline Image and Language & 1 \\
\hline 1. Consistency between image and content. & 0.67 \\
\hline 2. Language used for making this e-book. & 1 \\
\hline 3. The suitability of the picture presented. & 0.67 \\
\hline Alphabets, Background, and Color & 0.67 \\
\hline 1. Alphabet format used in the presentation. & 0.67 \\
\hline 2. Character size used. & 0.67 \\
\hline 3. Font color. & 1 \\
\hline 4. Background color. & 1 \\
\hline Electronics Media & \\
\hline 1. It is interesting to think and follow the lesson. \\
\hline 2. Presenting the e-book main title. \\
\hline 3. Presenting the e-book subtitle \\
\hline
\end{tabular}

The IOC points are more than 0.5 which means it is consistent and appropriate. So, the e-book was tried out with the undergraduate students who must learn astronomy.

In this study, 40 samples from both of the Chiang Mai Rajabhat University and Yala Rajabhat University were used for collecting data about satisfaction. The satisfaction analysis of the e-book for the basics of observing sky object is shown in Table III.

TABLE III. The EVAluation OF The E-Book By SAMPles

\begin{tabular}{|l|l|}
\hline \multicolumn{1}{|c|}{ Item } & \multicolumn{1}{|c|}{ evaluation } \\
\hline $\begin{array}{l}\text { 1. The e-book for the basics of } \\
\text { observing sky objects is interesting. }\end{array}$ & $4.75 \pm 0.44$ \\
\hline $\begin{array}{l}\text { 2. This e-book is not complex, easy } \\
\text { to use, not confused. }\end{array}$ & $4.78 \pm 0.42$ \\
\hline $\begin{array}{l}\text { 3. The user can understand and apply } \\
\text { to observation. }\end{array}$ & $4.88 \pm 0.23$ \\
\hline $\begin{array}{l}\text { 4. This e-book makes them want to } \\
\text { learn and want to research in the } \\
\text { future. }\end{array}$ & $4.85 \pm 0.36$ \\
\hline 5. Overall & $4.80 \pm 0.40$ \\
\hline
\end{tabular}

\section{CONCLUSIONS}

The e-book for the basics of observing sky objects was developed for undergraduate students. It was discovered from the analysis of the Astronomy description from many Rajabhat Universities. This e-book was improved the appropriate and consistent by using the IOC technique. It was tried out by the third-year students in general Science and Physics major at Chiang Mai Rajabhat University and Yala Rajabhat University. The satisfaction from 40 samples of the user is 4.80 . The most opinion is this e-book will be helping the user can understand and apply to observation.

In the future, we will adjust this e-book such as add some animation, connect to another website or database and expand from offline to online application.

\section{ACKNOWLEDGMENT}

The authors would like to thanks Chiang Mai Rajabhat University and Yala Rajabhat University, Thailand for supporting this work.

\section{REFERENCES}

[1] K. Panyasomsakool and W. Maithong, The Computer-Assisted Instruction Development on the Department of Astronomy and Space Telescope Secondary School. National Conference PSU, 2014.

[2] K. Kittiwachapong and W. Maithong, The Computer-Assisted Instruction Development about the Star in a Cause of the Earth, Astronomy and Space for the High School Students. National Conference PSU, 2014.

[3] S. Takaew, W. Maithong and S. Sairattnain, The Computer-Assisted Instruction on the Spaceship for Grade 9 Students. National Conference SSKRU, 2020.

[4] H. Karttunen, K. Kroger, H. Oja, M. Poutanen, and K. J. Donner, "Fundamental Astronomy," Germany: Springer - Verleg Berlin Heidelberg, 1987.

[5] S. Pattiyatanee, Educational Measurement (8th Ed.). Kalasin: Prasankanpim, 2012.

[6] S. Kanganawasee, Assessment Theory. Bangkok: Chulalongkorn University Press., 2007. 